\title{
Hack Harassment: Technology Solutions to Combat Online Harassment
}

\author{
George W. Kennedy III \\ Intel \\ Hillsboro, OR \\ george.w.kennedyeintel.com \\ Edward Dixon \\ Intel \\ Cork, Ireland \\ edward.dixon@intel.com \\ Chris Loo \\ Intel \\ Santa Clara, CA

\begin{abstract}
This work is part of a new initiative to use machine learning to identify online harassment in social media and comment streams. Online harassment goes underreported due to the reliance on humans to identify and report harassment, reporting that is further slowed by requirements to fill out forms providing context. In addition, the time for moderators to respond and apply human judgment can take days, but response times in terms of minutes are needed in the online context. Though some of the major social media companies have been doing proprietary work in automating the detection of harassment, there are few tools available for use by the public. In addition, the amount of labeled online harassment data and availability of cross platform online harassment datasets is limited. We present the methodology used to create a harassment dataset and classifier and the dataset used to help the system learn what harassment looks like.
\end{abstract}

\section{Introduction}

Online harassment has been a problem to a greater or lesser extent since the early days of the internet. Previous work has applied anti-spam techniques like machine learning based text classifica-

\author{
Andrew W. McCollough \\ EdgeRock Technology Partners \\ Hillsboro, OR
}

\author{
Alexie Bastidas \\ Intel \\ Santa Clara, CA
}

\author{
John Ryan \\ Intel \\ Cork, Ireland
}

\author{
Saurav Sahay \\ Intel \\ Santa Clara, CA
}

tion (Reynolds et al., 2011) to detecting harassing messages. However, existing public datasets are limited in size, with labels of varying quality.

The \#HackHarassment (Harassment, 2017) initiative (an alliance of tech companies and NGOs devoted to fighting bullying on the internet) has begun to address this issue by creating a web tool to collect and label data, and using the tool to generate a large, high-quality, cross-platform dataset. The release of this tool is scheduled for Summer 2017. As we complete further rounds of labelling with a public audience, later iterations of this dataset will increase the available samples by at least an order of magnitude and enable corresponding improvements in the quality of machine learning models we have built for harassment detection. In this paper, we introduce an improved cross-platform harassment dataset and a machine learning model built on the dataset.

\section{Related Work}

Previous work in the area by (Bayzick et al., 2011) showed that natural language processing in combination with a rule-based system could detect bullying messages on an online forum, but with very poor accuracy. However, the same work also made clear that the limiting factor on such models was the availability of a suitable quantity of labeled examples, e.g. the Bayzick work relied on a dataset of 2,696 samples, only 196 of which were 
found to be examples of bullying behavior. Additionally, this work relied on classical decision-tree models like J48 and JRIP, and k-nearest neighbors classifiers like IBk, as opposed to modern ensemble methods or deep neural-network-based approaches. In addition, Intel's \#HackHarassment team published work (Bastidas et al., 2016) showing results for harassment detection using a variety of model types on a new dataset of comments and posts which their team had labelled.

More recently, major internet companies have focused efforts on combating various forms of harassment online. Yahoo researchers have developed machine learning models for detecting abusive language (Nobata et al., 2016) and a Google Jigsaw team partnered with the Wikimedia Foundation to develop solutions for reducing personal attacks or toxic comments, in Wikimedia editing (Wulczyn et al., 2017). Nobata outperformed state-of-the-art deep learning approaches with their supervised learning approach using a combination of linguistic, n-gram (including character n-grams), syntactic (POS), and semantic (using comment embeddings similar to word2vec) features. In addition, the Yahoo team has released the longitudinal New Feed data set used in the study on (Webscope, 2017). Wulczyn demonstrated that their machine models can perform as well as three human graders in identifying toxic comments in Wikipedia editing wars, and in addition released the Perspective API to enable developers to utilize their solution. However, see (Hosseini et al., 2017) for comments on adversarial attacks and the resultant fragility of the model - and other models that depend on token-level features. We extend these results and others by developing a system architecture for crowdsourcing sample labeling, a crosssocial-media-platform dataset, and providing an open source classifier for developers to build upon. The classifier is intended to be open sourced in Summer 2017.

\section{Methods}

In this work, we build upon our initial results using version 1.0 of our dataset (Bastidas et al., 2016). We followed a supervised classification method that uses a data with gold-standard labeled comments and a set discriminating linguistic properties, or features, of each comment to predict the class membership of new or untrained comments. Our features consisted primarily of n-gram and a small set of linguistic features on datasets drawn from The Guardian, Reddit, and Twitter. We performed no significant pre-processing on the data other than tokenization, though in the future we anticipate adding further feature-reduction steps, such as stemming, to improve model performance.

\section{Data Source Selection}

Three initial data sources were selected: The Guardian, Reddit, and Twitter. Text from each data source were extracted in several ways in Summer 2016. Comments on polarizing or hot-button news articles were extracted from the Guardian, an online news source. Comments from Reddit, a popular social media site, were selected from comment which had received at least 100 down votes. Short texts from Twitter, tweets were hand-curated from an initially machine-selected data set from Twitter, and then further tweets scraped by searching on polarizing or hot-button topics.

\subsection{Reddit}

Comments from Reddit were downloaded from a publicly available dataset on Google BigQuery, reddit_comments_all_2015. These comments were then filtered to those that had received at least 100 down votes. We used our initial version of the classifier to label these comments. The resulting 5700 harassing comments were then further manually labeled by an in-house team of analysts. Analysts were given instructions and examples for annotation of harassment or non-harassment. In addition, the raters were provided with an additional set of more fine-grained labels but instruction on annotation was not provided.). Each post was labeled independently by at least five Intel Security Web Analysts. A perfect consensus was relatively rare, and so we rated a post as harassing if $40 \%, 2$ of our 5 raters, consider it to be harassing.

\subsection{Twitter}

Data were comprised of two sources: manual curation and annotation of a pre-existing machineannotated dataset and a set of scraped tweets using proprietary sampling methods. The sampling should not be considered unbiased. The initial 5000 tweets were sourced from an online repository of tweets at. Additional tweets were scraped directly from Twitter during July 2016 using a custom twitterbot that queried on hot-button topics as keywords to the Twitter API. These additional 


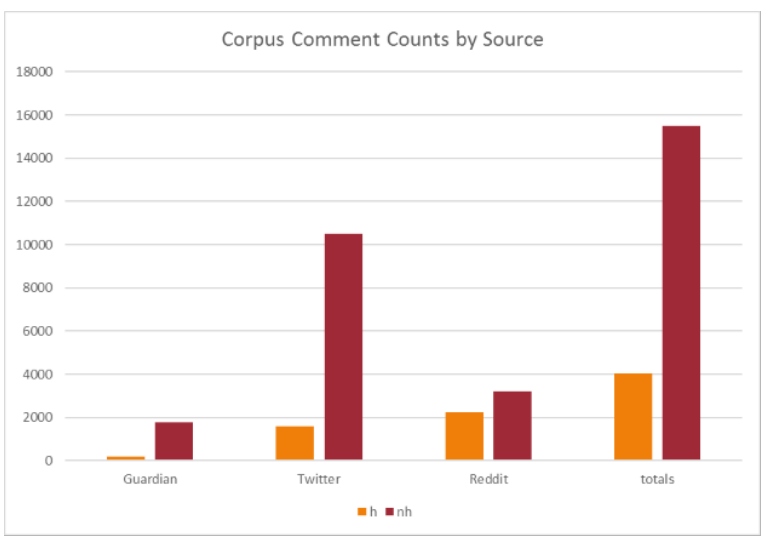

Figure 1: Corpus Comment Counts by Source

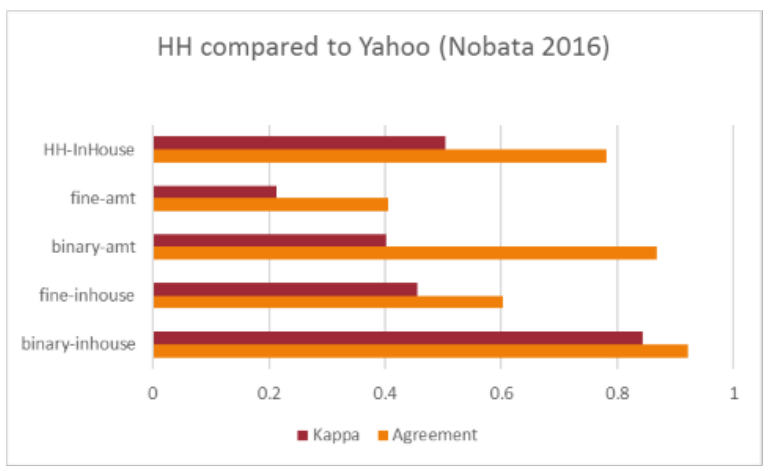

Figure 2: Inter-annotator agreement for Hack Harassment $(\mathrm{HH})$ compared to Yahoo. HH uses Krippendorfs Alpha and Yahoo uses Kappa. Agreement is an average pairwise agreement.

tweets were first labeled by our early classifier and then manually labeled by our team (Hart, 2016).

\subsection{The Guardian}

Comments were scraped from 15 articles covering hot-button or polarizing topics. We believe that minimal harassing comments were found in the Guardian dataset as Guardian comments are curated by a team of moderators in accordance with their content policy. Therefore, minimal or no harassing comments should be expected, as we confirmed in the dataset.

Figure 1 shows that the current data set is reasonably unbalanced overall with a $1: 4$ ratio of non-harassing to harassing comments. In addition, the categories are unbalanced across source as well as category within source, such that Reddit, despite being only $28 \%$ of the total comments contributed $56 \%$ of the harassing comments.

As shown in Figure 2 and Figure 3, average agreement is below $90 \%$ for the Guardian

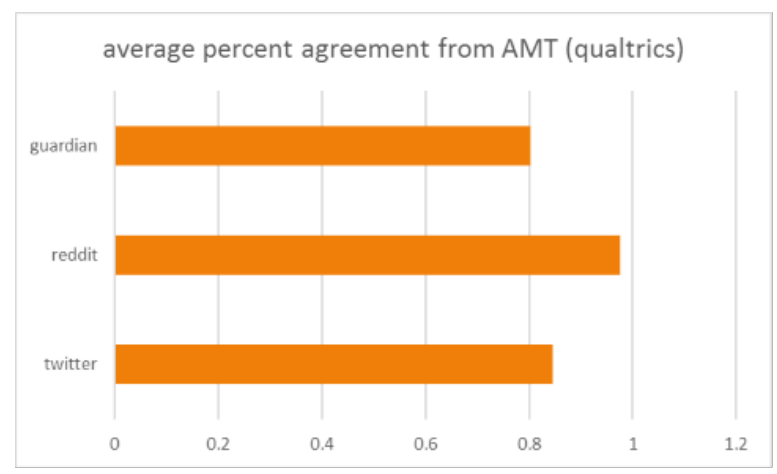

Figure 3: Average Percent of Agreement Among Amazon Mechanical Turk (AMT) Annotators

and Twitter surveys, with an average across all Qualtrics surveys only .875. This is well below what is typically suggested for raw agreement scores.

\begin{tabular}{|l|}
\hline Guardian URLs \\
\hline https://www.theguardian.com/discussion/p/4pcq2 \\
https://www.theguardian.com/discussion/p/4pgek \\
https://www.theguardian.com/discussion/p/4an9q \\
https://www.theguardian.com/discussion/p/4p76x \\
https://www.theguardian.com/discussion/p/4pdqd \\
https://www.theguardian.com/discussion/p/4phck \\
https://www.theguardian.com/discussion/p/4pf70 \\
https://www.theguardian.com/discussion/p/4pfe3 \\
https://www.theguardian.com/discussion/p/4k4tx \\
https://www.theguardian.com/discussion/p/4pd76 \\
https://www.theguardian.com/discussion/p/4jmg2 \\
https://www.theguardian.com/discussion/p/4pg57 \\
https://www.theguardian.com/discussion/p/4p6dt \\
https://www.theguardian.com/discussion/p/4p6gn \\
https://www.theguardian.com/discussion/p/4pgbx \\
\hline
\end{tabular}

Table 1: Guardian URLs used to scrape initial comments.

\section{Data Ingest and Annotation Methods}

Data ingest process and annotation were heterogeneous in nature. Manual curation was combined with machine annotation in several iterated steps to produce a final annotated dataset. The comment dataset was simply annotated with a Boolean indicating harassment. Harassment was determined on the gold data through a percent voting method: the reported metrics are for $40 \%$ and above simple agreement among raters that a given comment is harassment.

All preprocessing, training and evaluation was carried out in Python, using the popular SciKitLearn (for feature engineering and linear models) in combination with Numpy3 (for matrix operations) (Pedregosa et al., 2011; van der Walt et al., 
2011).

\section{Feature Selection}

Features were generated by tokenizing each comment, hashing the resulting n-grams, and computing a TF/IDF value for each token. The resultant feature vectors were used to train a Random Forest classifier. We used the following features:

- Unigram and Bigram TF-IDF: this is a standard feature used in text-categorization. We used unigrams and bigrams. Trigrams were not used because the size of the dataset meant almost all trigrams were too rare for their presence and absence to reach statistical significance.

- Character N-Gram TF-IDF from 3 to 6 characters: The goal with this was to target common alternative spellings of words, particularly frequent in online communication.

- Unigram Token Count: we utilized NLTKs Twitter Tokenizer to tokenize the tokens and count the number of tokens. The Twitter Tokenizer handles URLs and Hashtags much better than a standard punctuation based tokenizers found in NLTK or Sck-kit Learn. Our assumption behind using token count is that harassing texts tend to be brief assaults rather than long diatribes.

- Source: In combination with the token count, we selected a dummy coefficient (toggled as 1 or 0 ) to highlight if a comment is sourced from Twitter or not.

- Sentiment Polarities: we utilized NLTKs VADER Sentiment Analyzer to generate sentiment polarities for positive, neutral, and negative sentiment. Our assumption was that harassing comments tend to have more negative sentiment, whereas non-harassing comments tend to have more positive sentiment.

\section{Training Dataset}

The current training dataset contains: 20,432 unique comments. Of these comments, 4136 are labeled as harassment, 16296 are labeled as nonharassment. 12,049 comments are sourced from Twitter, with the remaining 8383 being from Reddit or the Guardian.

\section{Machine Learning Model}

Bastidas tested a variety of algorithms, including SVM, Decision Tree, Random Forest (ensemble Decision Trees), and Multinomial Nave Bayes (Bastidas et al., 2016). We increased the size of the Reddit dataset and included labeled comments that were sampled from Twitter and The Guardian. We collected performance results using this larger, cross-platform dataset, described in the Data Source Selection section, and a Scikit-Learn Random Forest classifier. For our hyperparameters we limited the number of trees to 200 and left the tree depth unbounded. Subsequently, the data were trained on the Random Forest by splitting the dataset into 80/20 training and evaluation sets, and then the training data were further split into Kfold $(n=10)$ folds for cross-validation and the average results reported in Table 2. Of primary concern to us is to optimize for high recall. We want to minimize our false-negative rate for harassment.

\begin{tabular}{|l|l|l|l|}
\hline Class & Precision & Recall & F1 Score \\
\hline Not Harassing & 0.93 & 0.95 & 0.94 \\
Harassing & 0.75 & 0.68 & 0.71 \\
Average & 0.89 & 0.90 & 0.90 \\
\hline
\end{tabular}

Table 2: Random forest classifier results.

\section{Future Work}

New work from Facebook and OpenAI on text classification suggests obvious next steps. Bytelevel deep neural nets are capable of state-oftheart results on large datasets, can exploit unlabeled data, as described in recent work from OpenAI (Radford et al., 2017) and have the potential to resist the "adversarial" tokens described in (Hosseini et al., 2017). Using OpenAI's approach with a large, unlabeled dataset for pre-training is an obvious next step. A contrasting approach that requires further evaluation is the FastText model from Facebook's Advanced Research Lab, which, as described in (Joulin et al., 2016) and (Bojanowski et al., 2016), is competitive with deep convolutional neural networks and can exploit unlabeled data using pre-trained WordVectors, while requiring vastly less training time than competitive alternatives.

\section{Conclusion}

We have presented to our cross-platform harassment dataset, machine learning model. We intend 
to open our labeling platform to the public to expand the Hack Harassment cross platform dataset. As we complete further rounds of labelling with a public audience, later iterations of this dataset will increase the available samples by at least an order of magnitude, enabling corresponding improvements in the quality of machine learning models for harassment detection. We look forward to both the availability of a larger, cross-socialmediaplatform harassment dataset and seeing the development of classifiers that improve upon our work. We welcome partners able to contribute to expanding the dataset and improving the modeling.

\section{References}

A. Bastidas, E. Dixon, C. Loo, and J. Ryan. 2016. Harassment detection: a benchmark on the hackharassment dataset. In Proceedings of the Collaborative European Research Conference (CERC 2016), Cork. pages 76-79.

J. Bayzick, A. Kontostathis, and L. Edwards. 2011. Detecting the presence of cyberbullying using computer software.

P. Bojanowski, E. Grave, A. Joulin, and T. Mikolov. 2016. Enriching word vectors with subword information. arXiv.org.

Hack Harassment. 2017. Hack harassment. https : //www. hackharassment. com/.

M. Hart. 2016. Twitterclassifier. https: //github.com/HackHarassment/ Twitterclassifier.

H. Hosseini, S. Kannan, B. Zhang, and R. Poovendran. 2017. Deceiving google's perspective api built for detecting toxic comments. arXiv. org.

A. Joulin, E. Grave, P. Bojanowski, and Mikolov. T. 2016. Bag of tricks for efficient text classification. arXiv.org.

Chikashi Nobata, Joel Tetreault, Achint Thomas, Yashar Mehdad, and Yi Chang. 2016. Abusive language detection in online user content. In Proceedings of the 25th International Conference on World Wide Web. International World Wide Web Conferences Steering Committee, Republic and Canton of Geneva, Switzerland, WWW'16, pages 145-153. https://doi.org/10.1145/2872427.2883062.

F. Pedregosa, G. Varoquaux, A. Gramfort, V. Michel, B. Thirion, O. Grisel, and et al. 2011. Scikit-learn: Machine learning in python. Journal of Machine Learning Research 12:145-153.
A. Radford, R. Jozefowicz, and I. Sutskever. 2017. Learning to generate reviews and discovering sentiment.

K. Reynolds, A. Kontostathis, and L. Edwards. 2011. Using machine learning to detect cyberbullying. In Presented at the 2011 Tenth International Conference on Machine Learning and Applications(ICMLA 2011), IEEE. pages 241-244.

S. van der Walt, S. C. Colbert, and G. Varoquaux. 2011. The numpy array: A structure for efficient numerical computation. Computing in Science \& Engineering 13(2):22-30.

Webscope. 2017. Webscope. https: //webscope.sandbox.yahoo.com/.

E. Wulczyn, D. Taraborelli, N. Thain, and L Dixon. 2017. Algorithms and insults: Scaling up our understanding of harassment on wikipedia. https://medium.com/jigsaw/algorithms-and-insultsscaling-up-ourunderstanding-of-harassment-onwikipedia-6cc417b9f7ff. 